\title{
Quantitative infrared thermography application for thermal defectometry
}

\author{
by V.A. STOROZHENKO (*)
}

(") Kharkov Institute of Radio Electronics, the Ukraine, 310726, Kharkov, Lenin Avenue, 14.

\begin{abstract}
A new method is described to solve the inverse problem of active thermal non destructive testing. This method allows to establish in an analytical form an interconnection between the defect parameters and the temperature response of the object. The possibility to define four defect parameters such as thickness, depth location, heat conductivity and diffusivity is shown.
\end{abstract}

\section{Nomenclature}

\begin{tabular}{|c|c|}
\hline & time, $[\mathrm{s}]$ \\
\hline$T$ & absolute temperature, [K] \\
\hline$\Delta T$ & exess temperature signal upon defect, [K] \\
\hline $\begin{array}{l}T(s), \hat{T} \\
A\end{array}$ & $\begin{array}{l}\text { Laplase and Fourier transforms of } T(\tau) \text {, respectively } \\
\text { temperature contrast upon defect }\end{array}$ \\
\hline$a$ & diffusivity, $\left[\mathrm{m}^{2} / \mathrm{s}\right]$ \\
\hline$d$ & layer thickness, [m] \\
\hline$x, y$ & space coordinates, $[\mathrm{m}]$ \\
\hline$H_{x}, H_{y}$ & cross-sectional dimension of defect, [m] \\
\hline & th of defect, [m] \\
\hline & spatial transter function of a layer \\
\hline$P$ & auxiliary transfer function of an object \\
\hline$Q$ & heat flux density, $\left[\mathrm{W} / \mathrm{m}^{2}\right]$ \\
\hline & heating power density, $\left[\mathrm{W} / \mathrm{m}^{2}\right]$ \\
\hline $\begin{array}{l}q(s), \hat{q} \\
R\end{array}$ & $\begin{array}{l}\text { Laplase and Fourier transforms of } q(\tau) \text {, respectively } \\
\text { heat transfer function of an object }\end{array}$ \\
\hline$\delta$ & defect thickness, [m] \\
\hline , & heat conductivity, $[\mathrm{W} / \mathrm{K} \mathrm{m}]$ \\
\hline$\tau_{h}$ & heating time, $[\mathrm{s}]$ \\
\hline d & time when $\Delta T$ or $A$ \\
\hline & onvection intensity, [ \\
\hline
\end{tabular}

\section{Essence of the problem}

Thermal defectometry is a new trend in the thermal non-destructive testing. Unlike a well known thermal defectoscopy the objective of the defectometry consists not only in a defect detection but also in its identification and its parameter definition. Modern infrared thermography facilities are well suited for thermal defectoscopy as they allow not only to obtain a temperature field image on the object surface but also to define by the embedded computing facilities quantitative characteristics of the function $\Delta T(x, y, \tau)$ such as an amplitude, location, area, etc.

But the defectometry needs special algorithms and programs for experimental data processing based on the solution for the inverse problem of non-stationary heat conduction. 


\section{http://dx.doi.org/10.21611/qirt.1992.044}

The essence of these algorithms and programs consists in establishing unambiguous correspondence between the measured function $\Delta T(x, y, \tau)$ and the parameters of the hidden defect being defined: $h, H_{x}, H_{y}, \delta, \lambda, a$.

Moreover, in the thermal defectometry a signal-to-noise ratio value is of particular concern, a thorough optimization of measurement conditions is needed for its increase.

Two above mentioned problems - defectometry algorithms construction and optimization of measurement conditions - are studied not to full extent for the time being.

Difficulties of the first problem are due to the non-stationary heat conduction inverse problem, being its basis, which is solved in the majority of publications on thermal defectometry either by the iteration numerical method [1] or by the analytical method of approximations [2], this reduces the degree of indormation in thermal defectometry and limits the speed of response. In particular, application of the indicated methods to solve the inverse problem allows to define only two parameters of the defect $-h$ and $\delta$ - of six mentioned above.

The second problem - optimization of measurement conditions characterized mainly by $\tau_{h}, q, \tau_{d}$ - is not given an adequate attention. Meanwhile the approach to optimization is known in the defectoscopy [3] and evidently can be used in the defectometry. Contents and volume of information selected from the bulk of the experimental data also needs optimization.

To develop the thermal defectometry further a search of new methods for the solution of the inverse problem of non-stationary heat conduction is necessary. To their number we can attribute a transfer function (TF) method less known in thermal physics where the object temperature response to the external action is regarded in terms of the product [4]

$$
T(s)=R q(s)
$$

$R$ is a heat transfer function.

The essence of the TF method organically combines with the defectometry contents as being defined from (1) $R$ is related to parameters of the object internal inhomogeneities defects.

This consideration predetermined the object of the present investigations - development of new approach to the solution of the inverse problem of non-stationary heat conduction on the TF method basis together with the optimization of thermal defectometry conditions and the process of data accumulation on the same basis.

\section{Theoretical model development}

Optimization of the thermal defectometry conditions consists in defining the above mentioned parameters $\tau_{h}, \tau_{d}$ and $q$ ensuring maximum signal-to-noise ratio for the considered volume. In general, the object is simulated by a plate (thickness I) with known thermophysical characteristics (TPC), and a defect is simulated by a rectangular parallelepiped [3].

The optimization process is realized through computation on the basis of the solution of the primal problem of non-stationary heat conduction. The condition of maximum of $\Delta T$ or $A$ is the optimization criterion. As a rule, the primal problem is solved by the mesh-point method. But the TF method, mentioned above, proves to be more exact and to have a fast response. The confirmation of this assumption is given below.

\subsection{Application of the TF method to solve the primal problem}

Let us analyse a n-layer unbounded plate whose thickness equals to I, one of its layers differing in TPC represents a defect, as a themophysical model of the object.

The mathematical model includes one-dimensional equation of non-stationary heat conduction with conventional boundary conditions $(\mathrm{BC})$ well-known in the thermal control theory. 


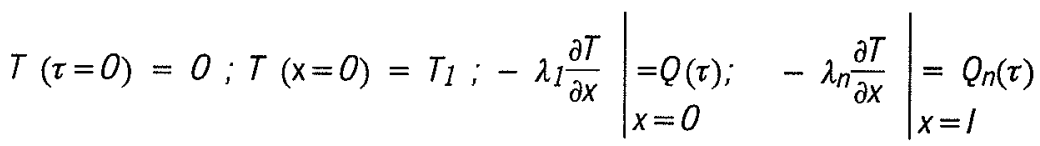

where $Q_{1}=q_{1}-\alpha_{1} T_{1} ; \quad Q_{n}=-q_{n}+\alpha_{n} T_{n}$.

We shall define the thermal transfer function $R$ [1] on the basis of matrix representation:

$$
\left|\begin{array}{l}
\hat{T}_{1} \\
\hat{T}_{n}
\end{array}\right|=R\left|\begin{array}{l}
\hat{q}_{1} \\
\hat{q}_{n}
\end{array}\right|=\left|\begin{array}{ll}
R_{11} & R_{12} \\
R_{21} & R_{22}
\end{array}\right| \cdot\left|\begin{array}{l}
\hat{q}_{1} \\
\hat{q}_{n}
\end{array}\right|
$$

where $\hat{T}_{1}, \hat{T}_{n}, \hat{q}_{1}, \hat{q}_{n}$ are Fourier image $T_{1}(\tau), T_{n}(\tau), q_{1}(\tau), q_{n}(\tau)$ functions, respectively.

To establish an interrelation between $R$ and the defect parameters $h, \delta, a_{d}, \lambda_{d}$, let us introduce the auxiliary $T F$ of the multilayer plate $P$, in significance this TF corresponds to the concept the spatial $T F$ and links thermal parameters $T$ and $q$ (their Fourier images) on the opposite sides of the plate:

$$
\left|\begin{array}{l}
\hat{T}_{n} \\
\hat{q}_{n}
\end{array}\right|=P\left|\begin{array}{l}
\hat{T}_{1} \\
\hat{q}_{1}
\end{array}\right|=\left|\begin{array}{ll}
P_{11} & P_{12} \\
P_{21} & P_{22}
\end{array}\right| \cdot\left|\begin{array}{l}
\hat{T}_{1} \\
\hat{q}_{1}
\end{array}\right|
$$

To obtain the spatial TF of the $n$-layer plate, the known solution of the equation for heat conduction with a substitution of the $\mathrm{BC}$ of (2) type should be written in the matrix form in the Fourier image domain. We shall obtain the spatial TF of one layer of the plate $J$

$$
\left|\begin{array}{l}
\hat{T}_{n} \\
\hat{Q}_{n} \\
k \lambda
\end{array}\right|=\left|\begin{array}{cc}
\cos k d & -\sin k d \\
\sin k d & \cos k d
\end{array}\right| \cdot\left|\begin{array}{c}
\hat{T}_{1} \\
\hat{Q}_{n} \\
k \lambda
\end{array}\right|=J\left|\begin{array}{l}
\hat{T}_{1} \\
\hat{Q}_{n} \\
k \lambda
\end{array}\right|,
$$

where $k=\sqrt{-i \omega / a}$.

Having introduced the conjugate matrix $[\mu]$ on the basis of the known continuity conditions on the layers boundary.

$$
\left[\mu_{1, i+1}\right]=\left|\begin{array}{ll}
1 & 0 \\
0 & \frac{\lambda_{i+1}}{\lambda_{i}} \sqrt{\frac{\alpha_{i}}{\alpha_{i+1}}}
\end{array}\right|
$$

we shall present TF of the n-layer plate as the product of TF of its separate layers $J_{i}$ and the conjugate matrices $[\mu]$. In this case, to single $\alpha$ out of $Q$ being a member of $(6)$, let us introduce a matrix $[\alpha]$ having for every external surface the form

$$
\left[\alpha_{1}\right]=\left|\begin{array}{ll}
1 & 0 \\
\alpha_{1} & . k \lambda
\end{array}\right| ; \quad\left[\dot{\alpha}_{n}\right]=\left|\begin{array}{cc}
1 & 0 \\
-\alpha_{n} & k \lambda
\end{array}\right|,
$$

As a result we shall obtain the expression for the spatial TF of a n-layer plate: 


$$
P=\left[\alpha_{n}\right] J_{n}\left[\mu_{n}, n+1\right] \ldots J_{i}\left[\mu_{i}, i+1\right] \ldots J_{1}\left[\alpha_{1}\right]-1
$$

The practical value of the obtained results consists in establishing the universal link between the thermal action $q$ and the temperature response $T$ which can be presented as a computer program and allows to analyse all possible regimes of the thermal control on the model prepared in advance.

\subsection{Inverse problem solution by the TF method}

From the equations (6) - (8) it is clear that the defect parameters $h, \delta, \alpha_{d}, \lambda_{d}$ form part of the TFs $R\left(h, \delta\right.$ are readily expressed in terms of $\left.d_{i}\right)$ which is linked according to (1) with: the thermal action on the object and the temperature response. Hence, there are preconditions for the solution of the inverse problem - establishment of interconnection of the defect parameters with $T$ and $q$ data being measured in defectometry.

It is clear that such a link can be established for the finite number of $n$ layers. Having assumed $n=3$, as it had been done in other publications on defectometry $[1,4]$, we obtained a system of three complex equations not presented here due to its awkwardness.

Taking into account that such a system is equivalent to six algebraic equations it is possible to conclude that the TF method widens the possibilities of thermal defectometry, it allows to find not only the defect geometric parameters $h$ and $\delta$ but its TPC: $a_{d}$ and $\lambda_{d}$.

Analyses of the obtained equations have led to an unexpected result it has turned out that the TF method allows to establish the presence of a defect on the preliminary stage without solving the problem to the end. Without defect $\left(d_{2}=0\right.$ or $\left.\mu=1\right)$ the left side of one of the equations becomes zero. This allows to introduce the defect presence criterion $\mathrm{K}$ :

$$
\begin{gathered}
K=\left[P_{11} \frac{\alpha_{n}}{k \lambda}+P_{22} \frac{\alpha_{1}}{k \lambda}+P_{21} \frac{1}{k \lambda}+P_{12}\left(\frac{\alpha_{1} \alpha_{n}}{k \lambda}+k \lambda\right)\right]^{2}+ \\
{\left[P_{11}-P_{22}+P_{12}\left(\alpha_{1}+\alpha_{n}\right)\right]^{2}}
\end{gathered}
$$

whose preliminary computation allows to establish the presence of a defect from the condition $K \neq 0$.

Solution of the equation system by numerical-analytical methods (one of the equations is transcendental) allows to express parameters of the defect through the elements of $R$ matrix which in their turn are linked according to (4) with the defectometry source data, i.e. $T$ and $q$

$$
\begin{aligned}
& R_{11}=\hat{T}_{1} / \hat{q}_{1} \\
& R_{21}=\hat{T}_{n} / \hat{q}_{1}
\end{aligned}\left|\begin{array}{l}
R_{12}=\hat{T}_{1} / \hat{q}_{n} \\
q_{n}=0
\end{array} R_{22}=\hat{T}_{n} / \hat{q}_{n}\right|_{q_{1}=0}
$$

Hence, the inverse problem solution by the TF method can be regarded as complete.

As only four unknowns are defined from the system of six algebraic equations then with definite values of $R$ matrix elements the system can have no solution. It proved that such a variant corresponds to the known situation in the defectometry where a defect is on top of another defect (defect over defect), i.e. the possibility to establish such situations by the TF methods has been found, this could not be done by the methods known until now. 


\section{Practical estimate of results}

For implementation of the defectometry method proposed above, the necessity is obvious to apply computing means. In this connection a program Layer is composed on the basis of the obtained equations.

The Layer program has been tested under two aspects. First we have verified the reversibility of the primal and inverse heat conduction problem to confirm the correctness of the problem statement and its solution method. Multiple runs in a wide range of the object parameters have shown agreement of the computation results, the error being less than $0,1 \%$. Second, the computer experiment has been carried out to estimate the influence of the introduced source data errors on the precision of the defectometry results. On the stage of the new method treatment such approach seems to be more productive than the real experimental data use, as it allows to single out the factors stipulating the defectometry precision and to study their influence in a wide range of object parameters. Among the influencing factors we considered the errors of the object's thermal diffusivity $\delta a$, its heat conduction $\delta \lambda$ and surface temperature $\delta T$.

Significant influence of even slight errors of the source data on the accuracy of defectometry has been ascertained. The degree of influence depends also on the controled object type, the kind of of the influencing factor, the defect parameter being measured. The most vulnerable factor is the TPC of the defect, and the most influencing factor is the temperature error. As a measure to increase the stability of the proposed TF method it can be offered to use several harmonics of Fourier image in finding the heat TF of the object and not one as it has been described above.

The thermal defectometry procedure is offered on the basis of the developed programs Plate and Layer

\section{Conclusion}

On the basis of the TF method the relations connecting the desired parameters of the defect with the temperature response to the specified thermal action have been obtained. The proposed approach is implemented for an one-dimensional model of the object in the form of a multilayer plate by means of the program Layer. Its tests confirmed that the TF method has advantages for thermal defectometry with respect to the productivity and content of information. In particular, the possibility has been shown to define not only the defect geometrical parameters but its thermophysical characteristics, and also to identify the defect over defect situation.

\section{REFERENCES}

[1] DURRANI (T.S.), RAUF (A.) and BOGLE (K.), - Reconstruction techniques for the inspection of composite materials using thermal images. ICASSP'88: Int. Conf. Spr. a. Signal Process., N.Y., 1988, p. 863-866.

[2] VAVILOV (V.P.), AKHMED (T.) and GIPP C(H.D.).- Experimental Termography of solids in pulse one-side heating. Defectoscopy, 1990, N12, p. 60-66.

[3] STOROZHENKO (V.A.), VAVILOV (V.P.) and VOLCHEK (A.D.). - Non-destructive control of the industrial production quality by active thermal method. Kiev, « Tekhnika», 1988, - $87 \mathrm{p}$.

[4] KOKKAS (A.G.). -Thermal analysis of multiple-layer structures. IEEE Trans. Electr. Devices, 1974, N11, p. 674-681. 
http://dx.doi.org/10.21611/qirt.1992.044 